\title{
DESENVOLVIMENTO DE UM DISPOSITIVO ELETROSTÁTICO PARA PULVERIZADOR PNEUMÁTICO COSTAL
}

\author{
Ronaldo Goulart Magno Júnior ${ }^{1}$, Mauri Martins Teixeira², Haroldo Carlos Fernandes ${ }^{3}$, Denílson Eduardo \\ Rodrigues $^{4}$, Cleyton Batista de Alvarenga ${ }^{5}$
}

\begin{abstract}
RESUMO
A pulverização eletrostática consiste no carregamento das gotas com cargas elétricas, criando-se um campo elétrico capaz de aumentar a atração das gotas pelas plantas. Objetivou-se com este trabalho desenvolver um dispositivo eletrostático para pulverizador pneumático costal, utilizando uma fonte de alta tensão e baixo custo. Utilizou-se uma bobina de ignição automotiva ligada a um circuito eletrônico alimentado por uma bateria de 12 volts. A fonte de alta tensão foi instalada na parte de trás do pulverizador e ligada ao dispositivo de eletrificação indireta, que foi construído de cobre, com 0,10 m de diâmetro e 0,03 m de espessura. A medição da corrente no jato de gotas foi realizada usandose uma Gaiola de Faraday, ligada a um micro-amperímetro. A fonte forneceu nove diferentes condições de trabalho, com tensões ajustáveis entre 4,2 e $12,8 \mathrm{kV}$, o que permitiu atingir valores de relação carga/massa de $0,3 \mathrm{mC} \mathrm{kg}^{-1}$ a 4,1 $\mathrm{mC} \mathrm{kg}{ }^{-1}$, trabalhando com vazão de 0,$6 ; 1,5$; e $1,9 \mathrm{~L} \mathrm{~min}^{-1}$.
\end{abstract}

Palavras-chave: pulverização eletrostática, agrotóxicos, vazão de liquido.

\section{ABSTRACT \\ DEVELOPMENT OF AN ELECTROSTATIC DEVICE FOR A HYDRAULIC BACKPACK SPRAYER}

Electrostatic spraying consists of charging droplets with electric charge, creating an electric field can increase the attraction of droplets by the plants. The objective of this study was to develop an electrostatic device for a hydraulic backpack sprayer, using a high voltage and low cost source. An automotive ignition coil was connected to an electronic circuit powered by a 12 volt battery. The high voltage supply was installed on the rear of the sprayer and connected to a indirect electrification device, which was constructed of copper, measuring $0.10 \mathrm{~m}$ in diameter and $0.03 \mathrm{~m}$ thick. Flow measurement in the spray nozzle was performed using a Faraday cage, connected to a micro-ammeter. The source provided nine different working conditions, with adjustable voltage between 4.2 and $12.8 \mathrm{kV}$, which allowed

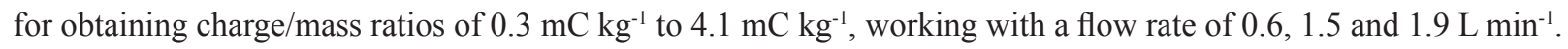

Keywords: electrostatic spraying, pesticides, liquid flow rate.

\section{Recebido para publicação em 09/03/2011. Aprovado em 01/02/2013.}

1 - Engenheiro Agrônomo, Professor, Instituto Federal de Minas Gerais, Bambuí-MG, Brasil, ronaldoufla@yahoo.com.br

2 - Engenheiro Agrônomo, Professor, UFV, Departamento de Engenharia Agrícola, Viçosa-MG, Brasil, mauri@ufv.br

3 - Engenheiro Agrícola, Professor, UFV, Departamento de Engenharia Agrícola, Viçosa-MG, Brasil, haroldo@ufv.br

4 - Engenheiro Agrícola, Professor, UFV, Departamento de Eng. Elétrica e de Produção, Viçosa-MG, Brasil, deduardo@ufv.br

5 - Engenheiro Agrônomo, Professor, UFOPA, Inst. de Biodiversidade e Florestas, Santarém-PA, Brasil, cleyton.alvarenga@ufopa.edu.br 


\section{INTRODUÇÃO}

A pulverização eletrostática consiste no carregamento das gotas com cargas positivas ou negativas, criando um campo elétrico e fazendo com que a gota não mude de trajetória entre o pulverizador e o alvo, sendo atraída por este (CHAIM, 2006). É justamente quando se produz gotas pequenas que forças elétricas podem ser induzidas em grandeza suficiente para controlar seu movimento, inclusive contra a gravidade, o que proporcionaria às gotas a capacidade de se depositarem na face inferior das folhas (CHAIM, 2004).

A deposição de gotas com cargas eletrostáticas pode ser até $70 \%$ superior à obtida com as gotas sem cargas, dependendo das características morfológicas do alvo e da própria intensidade de carga nas gotas (LAW; COOPER, 1988).

Chaim (2006) e Matthews (1989) descreveram três processos utilizados na eletrificação de gotas: eletrificação por efeito-corona, eletrificação por indução direta e eletrificação por indução indireta e, afirmaram que a eficiência da pulverização eletrostática é diretamente relacionada ao processo utilizado para eletrificar as gotas.

No método de eletrificação indireta, o líquido é mantido aterrado, sem tensão. Nesse processo, as gotas adquirem a carga na presença de intenso campo eletrostático, formado entre o eletrodo de indução $(1 \mathrm{a} 10 \mathrm{kV})$ e o jato de gotas. $\mathrm{O}$ eletrodo deve ser posicionado na região da borda do jato onde as gotas se formam, em uma distância mínima suficiente para evitar centelhas de descarga entre o eletrodo e o líquido. A vantagem desse método é o fato de que o líquido contido no tanque e nas tubulações fica submetido à voltagem zero, e necessita-se de tensões relativamente baixas para eletrificação das gotas.

Avaliando a deposição de agrotóxicos com bocal eletrostático, Chaim et al. (2002) verificaram que um aumento na tensão de indução aumenta a intensidade de carga das gotas até determinado limite, a partir do qual, incrementos na tensão são prejudiciais à eletrificação das partículas. Concluíram também que o aumento na carga das gotas aumenta a deposição da pulverização. Neste trabalho, usando uma tensão de $8 \mathrm{kV}$ e uma vazão de $0,3 \mathrm{~L} \mathrm{~min}^{-1}$, obteve-se uma carga máxima de $4,0 \mathrm{mC} \mathrm{kg}^{-1}$.
Kang et al. (2004) avaliando um pulverizador eletrostático pneumático, equipado com uma fonte de alta tensão pulsante, ajustável de 0 a 8 $\mathrm{kV}$ e alimentada por uma bateria de $12 \mathrm{~V}$ e 40 $\mathrm{A}$, verificaram que a tensão mais eficiente no processo de eletrificação de gotas foi de $4 \mathrm{kV}$. Afirmaram que em tensões acima de $4,5 \mathrm{kV}$ houve centelhamento entre o eletrodo e o bocal eletrostático.

Avaliando o efeito da tensão de indução sobre o jato de gotas do pulverizador, Maski (2005) obteve uma relação carga/massa de $18,5 \mathrm{mC} \mathrm{kg}^{-1}$ e 7,5 mC kg-1 , usando tensão de indução de 7,5 e $4,0 \mathrm{kV}$, respectivamente, com uma vazão de

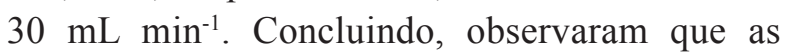
propriedades do líquido, como densidade, tensão superficial, condutividade elétrica e constante dielétrica, afetam o processo de eletrificação das gotas.

Maski et al. (2004), avaliando parâmetros que influenciam a indução eletrostática, concluíram que as propriedades do líquido e o método de eletrificação podem afetar o processo de formação das gotas, a capacidade de eletrificar as gotas, a carga máxima que pode acumular numa gota, o nível de carga, a velocidade terminal, a eficácia biológica e a deposição no alvo.

Em relação ao mercado, existe comercialmente o equipamento Spectrum Electrostatic Aerial Spray System que apresenta um sistema eletrostático que opera com alta pressão na barra de pulverização e baixo volume, carregando todas as partículas, principalmente em aeronaves, de forma bipolar, produzindo cargas com polaridades opostas em cada barra de pulverização. Seu grande diferencial, em relação à pulverização convencional, está nas gotas, com diâmetro de 150 $\mu \mathrm{m}$ que, depois de geradas pela energia hidráulica das pontas, são submetidas a um potente campo elétrico, posicionado ao seu redor, carregandoas eletricamente, de maneira que passam a ser fortemente atraídas pelas plantas (SCHRODER, 2006).

Objetivou-se com este trabalho construir um dispositivo eletrostático para um pulverizador pneumático de eletrificação indireta, capaz de gerar um nível de carga suficiente para aumentar a eficiência da aplicação de agrotóxicos. 


\section{MATERIAL E MÉTODOS}

A construção do gerador de cargas eletrostáticas e o desenvolvimento do dispositivo para o pulverizador foram realizados no laboratório de aplicação de agrotóxicos do Departamento de Engenharia Agrícola e no laboratório de eletrônica do Departamento de Engenharia Elétrica e de Produção, ambos da Universidade Federal de Viçosa.

Para desenvolver o dispositivo eletrostático, que foi adaptado a um pulverizador pneumático costal motorizado, da marca Yannmar, com tanque de capacidade de $14 \mathrm{~L}$, motor de 1,3 HP, utilizouse uma fonte de alta tensão, geradora de cargas eletrostáticas, com tensão ajustável entre 4,0 e 12,8 $\mathrm{kV}$ e corrente elétrica de $40 \mu \mathrm{A}$, semelhante ao proposto por Celen et al. (2009).

A fonte de alta tensão foi composta por uma bobina automotiva de $12 \mathrm{~V}$, um circuito integrado modelo 555 (temporizador), um transistor modelo 2N3055, dois resistores, um potenciômetro para ajustar a variação de tensão, um capacitor eletrolítico, um capacitor de poliéster e dois diodos 1N4001, a alimentação foi feita por uma bateria de $12 \mathrm{~V}$.

Foram realizadas nove marcações no potenciômetro, a fim de variar a tensão e a corrente na fonte. Deste modo, cada marcação constituía um tratamento. Para medir a tensão da fonte, foi utilizada uma ponta de prova para alta tensão, com capacidade para $40 \mathrm{kV}$, semelhante ao utilizado por Serra et al. (2008) e acoplada a um multímetro digital. A corrente foi medida com auxílio de um micro-amperímetro. Para determinar a frequência de pulso e a forma da onda gerada pela fonte foi usado um osciloscópio modelo OS 9030.

A fonte foi acondicionada em uma caixa metálica, a fim de evitar interferência nos componentes eletrônicos oriundos do ruído do motor de acionamento do pulverizador.

A caixa metálica, funcionando como gaiola de Faraday, foi fixada na parte traseira do pulverizador (Figura 1). A saída da fonte de alta tensão foi conectada ao anel eletrificador, por meio de cabos de alta isolação, protegidos por mangueira, visando maior segurança.

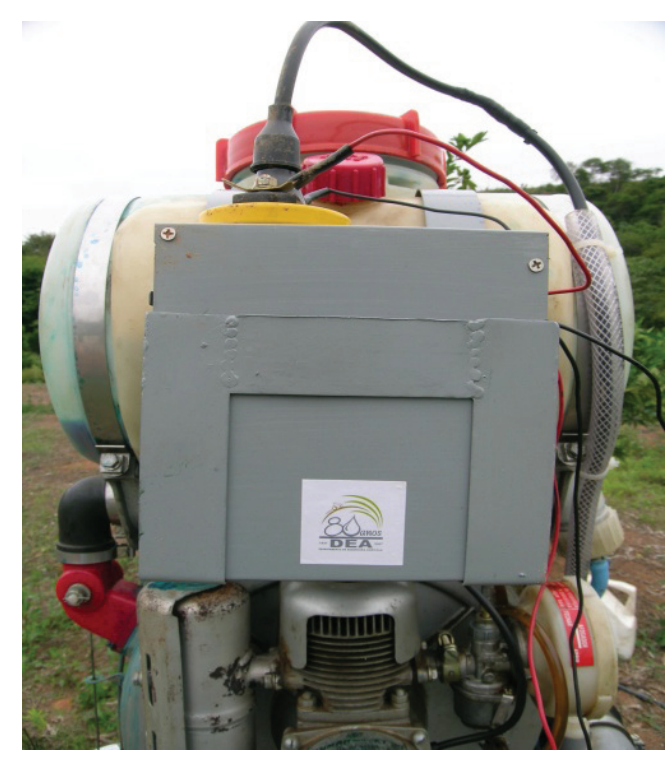

Figura 1. Fonte de alta tensão adaptada ao pulverizador.

Primeiramente, foi construído um dispositivo, semelhante a um anel de diâmetro ajustável, de alumínio. Posteriormente, utilizando-se o anel realizaram-se vários testes para verificar qual o diâmetro adequado para que o jato de gotas não atingisse o anel, evitando, assim, escorrimento de líquido. Nos ensaios preliminares, constatouse que diâmetros inferiores a $0,10 \mathrm{~m}$ causariam escorrimento de líquido devido ao contato do jato de gotas com o anel de indução. Também foram realizados testes para determinar a distância do anel de indução em relação à saída de líquido do pulverizador, determinando que a melhor distância fosse de $0,05 \mathrm{~m}$.

$\mathrm{O}$ anel foi construído em cobre, com $0,10 \mathrm{~m}$ de diâmetro e $0,03 \mathrm{~m}$ de largura, e fixado na lança do pulverizador por um material isolante (Figura 2).

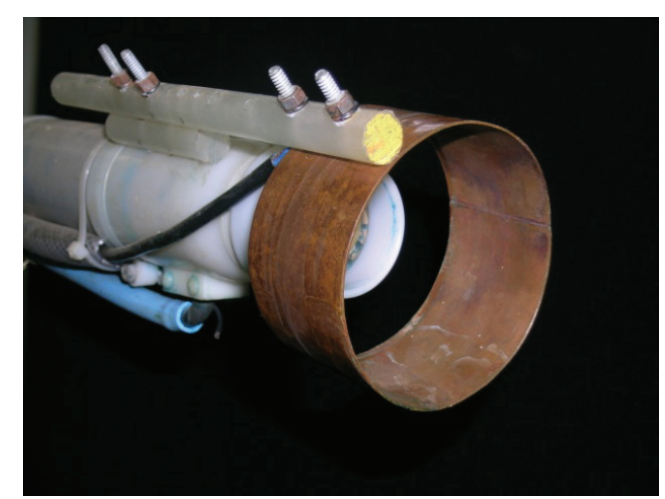

Figura 2. Dispositivo eletrostático em forma de anel de cobre para indução indireta. 
A quantidade de carga do sistema foi determinada de acordo com o método usado por Chaim (1998) e Simmons e Lehtinen (1986), que consiste no uso de uma gaiola de tela metálica (gaiola de Faraday), isolada e posicionada a $0,05 \mathrm{~m}$ de distância do bocal do pulverizador.

A gaiola foi aterrada por meio de uma haste metálica de $1,0 \mathrm{~m}$ de comprimento, sendo conectado ao circuito um multímetro, com escala de 0 a $50 \mu \mathrm{A}$, com sensibilidade para $1 \mu \mathrm{A}$. Dessa forma, determinou-se a descarga de eletricidade contida no jato de gotas. A relação carga/massa foi obtida pela relação entre a corrente elétrica $\left(\right.$ Coulomb s${ }^{-1}$ ) e a quantidade da massa de líquido pulverizada $\left(\mathrm{kg} \mathrm{s}^{-1}\right)$.

A força de atração, ou repulsão, exercida entre duas cargas elétricas concentradas em dois pontos distintos depende do meio em que se verifica o fenômeno e, em dado meio, é proporcional ao produto de suas massas e inversamente proporcional ao quadrado da distância que as separa (Equação 1).

$F=k \frac{q_{1} q_{2}}{d^{2}}$

em que,

F- força de Coulomb (N);

$\mathrm{K}$ - constante eletrostática, $\left(910^{9} \mathrm{~N} \mathrm{~m}^{2} \mathrm{C}^{-2}\right)$;

$\mathrm{q}_{1}$ e $\mathrm{q}_{2}$ - carga elétrica, (C);

d- distância entre duas cargas (m).

A partir dos valores obtidos na Equação 1, determinou-se o valor do campo elétrico (Equação 2).

$E=\frac{F}{q}$

em que,

E- campo elétrico $\left(\mathrm{N} \mathrm{C}^{-1}\right)$;

F- força elétrica $(\mathrm{N})$;

q- carga elétrica, (C).
A relação entre a carga eletrostática e a diferença de potencial é expressa da seguinte maneira (Equação 3).

$Q=V C$

em que,

Q- carga eletrostática no objeto (C);

$\mathrm{V}$ - diferença de potencial no sistema, (V);

C- capacitância (F).

As gotas menores são mais facilmente eletrificadas, pois atingem o Limite de Rayleigth com mais facilidade (Equação 4). A gota alcança esse limite quando ela acumula a máxima quantidade de carga possível.

$q=8 \pi \sqrt{\gamma \varepsilon_{0}} r^{3}$

em que,

q- carga na gota (C);

$\gamma$ - tensão superficial $\left(\mathrm{N} \mathrm{m}^{-1}\right)$;

$\varepsilon_{0}$ - permissividade do líquido $\left(\mathrm{C} \mathrm{N}^{-1} \mathrm{~m}^{2}\right)$;

r- raio da gota $(\mu \mathrm{m})$.

\section{RESULTADOS E DISCUSSÃO}

A fonte utilizada foi capaz de fornecer a tensão e a corrente necessária para eletrificação das gotas pulverizadas. Com a retificação da fonte de alta tensão, os níveis de corrente contidos no jato de pulverização foram muito baixos. Devido a esse fato, a fonte foi usada sem a retificação da onda, apenas com a polarização do sinal, feito por meio de diodos.

$O$ fato da onda retificada não ter eletrificado as gotas pode ser explicado baseando-se no conceito de Faraday, onde para ter corrente elétrica gerada por força eletromotriz, é preciso que haja um campo eletromagnético variável no tempo.

As características da fonte medidas em laboratório e o valor da corrente de acordo com a posição de regulagem do cursor do potenciômetro são apresentados no Quadro 1. 
Quadro 1. Valores de tensão, freqüência e corrente da fonte de alta tensão

\begin{tabular}{cccc}
\hline Tratamento & Tensão $(\mathrm{kV})$ & Freq. $(\mathrm{Hz})$ & Corrente $(\mu \mathrm{A})$ \\
\hline 1 & $4,20 \mathrm{a}$ & $1562,50 \mathrm{a}$ & $10,00 \mathrm{a}$ \\
2 & $6,03 \mathrm{~b}$ & $1191,18 \mathrm{~b}$ & $11,66 \mathrm{ab}$ \\
3 & $8,00 \mathrm{c}$ & $842,90 \mathrm{c}$ & $14,33 \mathrm{bc}$ \\
4 & $9,60 \mathrm{~d}$ & $647,00 \mathrm{~d}$ & $17,00 \mathrm{~cd}$ \\
5 & $10,13 \mathrm{de}$ & $600,91 \mathrm{de}$ & $20,33 \mathrm{~d}$ \\
6 & $10,93 \mathrm{e}$ & $543,47 \mathrm{def}$ & $24,00 \mathrm{e}$ \\
7 & $12,00 \mathrm{f}$ & $476,19 \mathrm{efg}$ & $28,00 \mathrm{f}$ \\
8 & $12,26 \mathrm{f}$ & $416,64 \mathrm{fg}$ & $38,00 \mathrm{~g}$ \\
9 & $12,80 \mathrm{f}$ & $394,76 \mathrm{~g}$ & $41,00 \mathrm{~g}$ \\
\hline
\end{tabular}

Médias seguidas de mesma letra não diferem a $5 \%$ de probabilidade, pelo teste de Tukey.

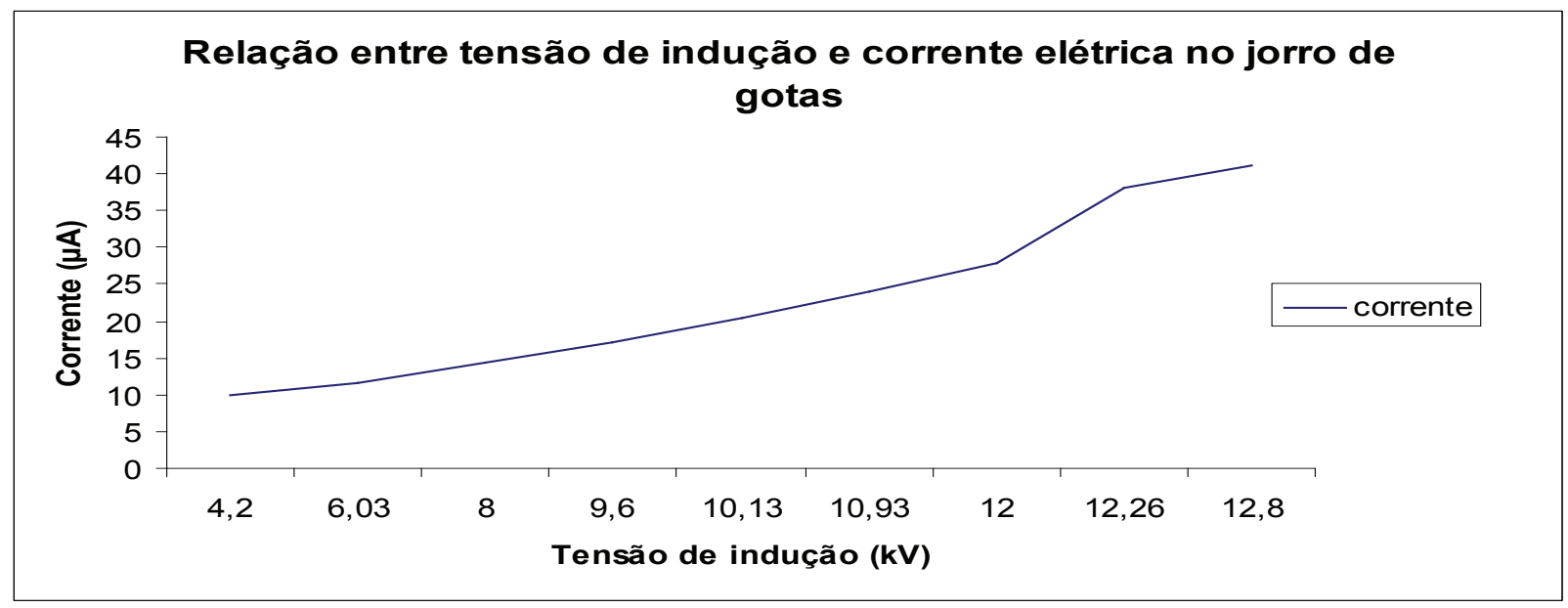

Figura 3. Relação entre tensão de indução e corrente elétrica no jato de gotas.

É possível observar que, com o aumento na posição do potenciômetro, consequentemente, ocorreu aumento nas tensões e correntes. Embora as tensões nas posições de 7 a 9 tenham sido estatisticamente iguais, apenas as correntes nas posições 8 e 9 são estatisticamente iguais entre si.

Na Figura 3 ilustra-se a elevação da corrente em função do incremento da tensão de indução. Observou-se um aumento mais acentuado de corrente entre as tensões de 12,00 e $12,26 \mathrm{kV}$, sendo o pico em $12,8 \mathrm{kV}$.

$\mathrm{O}$ dispositivo do pulverizador eletrostático desenvolvido forneceu relação carga/massa acima do nível mínimo citado por Carlton (1995), que é de $0,8 \mathrm{mC} \mathrm{kg}^{-1}$.

Nos ensaios de relação carga/massa, foram utilizadas as nove correntes disponíveis na fonte, além de três vazões: 0,$6 ; 1,5$ e $1,9 \mathrm{~L} \mathrm{~min}^{-1}$, com três repetições. No Quadro 2, apresentam-se os valores da relação carga/massa, nas diferentes vazões e diferentes correntes usadas no ensaio.

Conforme pôde ser analisado, no Quadro 2, com o aumento na vazão ocorreu redução significativa na relação carga/massa. Esse resultado pode ser explicado pelo incremento da massa pulverizada por minuto e também pelo tamanho das gotas geradas nas diferentes vazões. Aumentando a vazão do pulverizador, há aumento no tamanho de gotas, visto que a quantidade de ar para fragmentar maior massa de líquidos permanece constante, corroborando com os resultados apresentados por Chaim et al. (2002); Serra et al. (2008) e Almekinders et al. (1992). 
Quadro 2. Relação carga/massa $\left(\mathrm{mC} \mathrm{kg}^{-1}\right)$ em função da vazão e da corrente

\begin{tabular}{cccc}
\hline \multirow{2}{*}{ Tratamento } & \multicolumn{3}{c}{ Vazão $\left(\mathrm{L} \mathrm{min}^{-1}\right)$} \\
\cline { 2 - 4 } & 0,6 & 1,5 & 1,9 \\
\hline 2 & 1,00 & 0,40 & 0,31 \\
3 & 1,16 & 0,46 & 0,36 \\
4 & 1,43 & 0,57 & 0,45 \\
5 & 1,70 & 0,68 & 0,53 \\
6 & 2,03 & 0,81 & 0,64 \\
7 & 2,40 & 0,96 & 0,75 \\
8 & 2,80 & 1,52 & 1,20 \\
9 & 3,80 & 1,52 & 1,20 \\
\hline
\end{tabular}

Quadro 3. Valores da relação carga/massa em função da vazão

\begin{tabular}{cc}
\hline Vazão $\left(\mathrm{L} \min ^{-1}\right)$ & Médias $\left({\left.\mathrm{mC} \mathrm{kg}^{-1}\right)}^{-1}\right.$ \\
\hline 0,6 & $4,10 \mathrm{a}$ \\
1,5 & $1,64 \mathrm{~b}$ \\
1,9 & $1,29 \mathrm{~b}$ \\
\hline
\end{tabular}

Médias seguidas de mesma letra não diferem estatisticamente a $5 \%$ de probabilidade, pelo teste de Tukey.

A melhor relação $\mathrm{Q} / \mathrm{M}$ foi verificada na maior corrente disponível na fonte e na vazão de $0,6 \mathrm{~L} \mathrm{~min}^{-}$ 1, sendo significativamente diferente das vazões de 1,2 e $1,5 \mathrm{~L} \mathrm{~min}^{-1}$. Portanto, com essa vazão a velocidade de deslocamento do pulverizador teria que ser muito baixa para atingir um volume usual em nível de campo, em torno de $28 \mathrm{~L}$ por planta no caso dos citros (RAMOS et al., 2007).

No Quadro 3, apresenta-se a média dos níveis de carga gerados nas deferentes vazões para a máxima corrente da fonte $(41 \mu \mathrm{A})$.

Analisando-se os resultados apresentados no Quadro 3, pode-se verificar que a relação carga/ massa nas vazões de 1,5 e $1,9 \mathrm{~L} \min ^{-1}$ foram estatisticamente iguais. Embora aparentemente a média da relação carga/massa na vazão de 1,9 $\mathrm{L} \min ^{-1}$ fosse menor que a média de $1,5 \mathrm{~L} \mathrm{~min}^{-1}$, estatisticamente não houve diferença entre os dois valores e sim diferença estatística somente na vazão de $0,6 \mathrm{~L} \mathrm{~min}^{-1}$.

O nível de carga na vazão de $0,6 \mathrm{~L} \mathrm{~min}^{-1}$ pode ser considerado muito bom, mas essa vazão não seria recomendada para pulverização em plantas arbóreas. Uma vazão dessa proporção poderia ser usada em casa de vegetação, onde há ausência de vento, podendo-se trabalhar com uma vazão muito baixa e, consequentemente, com tamanhos de gotas muito pequenos.

$\mathrm{Na}$ Figura 4 está ilustrado o decréscimo da relação carga/massa em função do aumento da vazão do pulverizador. Esse decréscimo é devido ao aumento da massa de água, diminuindo, assim, a relação carga/massa. Resultados semelhantes foram obtidos por Chaim et al. (2002), com tensão de indução de $8 \mathrm{kV}$ e vazão variando de 0,1 a $0,8 \mathrm{~L}$ $\mathrm{min}^{-1}$, trabalhando com pulverizador equipado com dispositivo eletrostático em equipamento costal motorizado.

\section{REVENG}




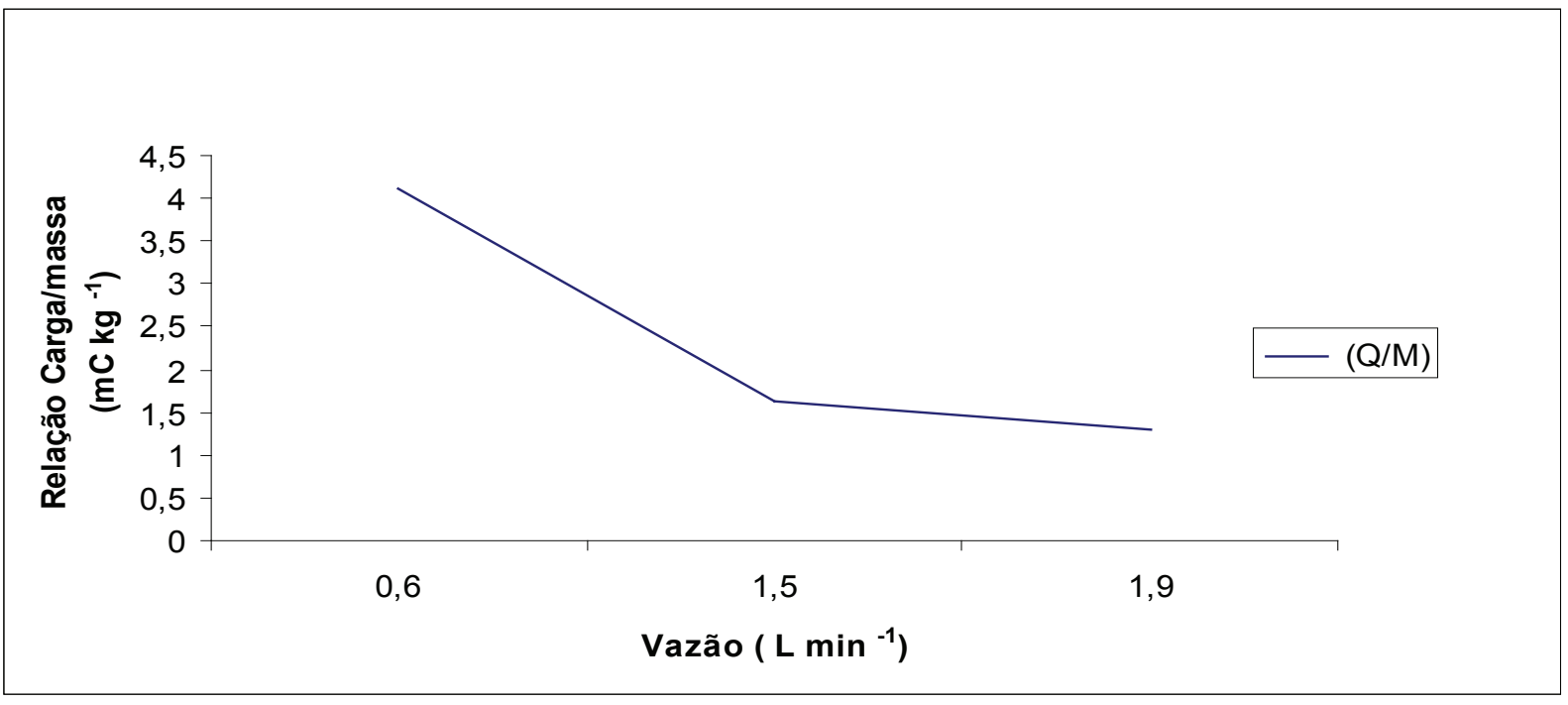

Figura 4. Relação entre intensidade de carga e vazão, com tensão de 12,8 kV.

Mesmo com o uso da pulverização eletrostática, em que a força de atração entre a gota e o alvo pode ser aumentada em até 40 vezes (LAW, 2001), o uso de volumes muito baixos em pulverizadores pneumáticos poderia causar menor cobertura, devido à deriva e evaporação, diminuindo a eficácia de controle e causando, consequentemente, resistência das pragas e doenças aos agrotóxicos. A pulverização eletrostática pode levar a uma redução no volume de calda aplicado, redução da contaminação ambiental e dos operadores. Resultados que corroboram com esta temática foram encontrados por Serra et al. (2008); Kabashima et al. (1995); Chaim et al. (2002); Schroder e Loeck, (2006).

\section{CONCLUSÕES}

- O dispositivo eletrostático acoplado ao pulverizador pneumático foi capaz de eletrificar as gotas pulverizadas;

- Os níveis de carga gerados pelo sistema eletrostático, na maior vazão e na maior tensão, foram superiores a $0,8 \mathrm{mC} \mathrm{kg}^{-1}$;

- À medida que aumentou a vazão do pulverizador houve decréscimo na relação carga/massa.

\section{REFERÊNCIAS BIBLIOGRÁFICAS}

ALMEKINDERS, H.; OZKAN, H.E.; REICHARD, D.L.; CARPENTER, T.G.; BRAZEE, R.D. Spray deposit patterns of an electrostatic atomizer. Transactions of the ASAE, v.35, p.1361-1367, 1992.

CELEN, I.H.; DURGUT, M.R.; AVCI, G.G.; KILIC, E. Effect of air assistance on deposition distribution on spraying by tunnel-type electrostatic sprayer. African Journal of Agricultural Research, v.4, n.12, p.1392-1397, 2009.

CHAIM, A. Aperfeiçoamento de bico de pulverização eletrostática para geração de gotas com alto nível de carga. Pesquisa Agropecuária Brasileira, Brasília, v.33, n.4, p.463-470, 1998.

CHAIM, A.; PESSOA, M.C.P.Y.; FERRACINI, V. Eficiência de deposição de agrotóxicos, obtida com bocal eletrostático para pulverizador motorizado costal motorizado. Pesquisa Agropecuária Brasileira, Brasília, v.37, n.4, p.497-501, 2002.

CHAIM, A. Pulverização eletrostática. Qualidade em tecnologia de aplicação. Botucatu, SP: UNESP, 2004. p.85-92. 
CHAIM, A. Pulverização eletrostática: principais processos utilizados para eletrificação de gotas. Jaguariúna, SP: Embrapa Meio Ambiente, 2006. 18p. (Documento 57).

KABASHIMA, J.; GILES, D.K.; PARRELLA, M.P. Electrostatic sprayers improve pesticide efficacy in greenhouses. California Agriculture, v.49, p.31-35, 1995.

KANG, T.G.; LEE, D.H.; LEE, C.S.; KIM, S.H.; LEE, G.I.; CHOI, W.K.; NO, S.Y. Spray and deposition characteristics of electrostatic nozzles for orchard sprayers. St. Joseph, Mich: ASAE, 2004. (Paper $n^{\circ}$ 041005).

LAW, S.E.; COOPER, S.C. Depositional characteristics of charged and uncharged droplets applied by an orchard air carrier sprayer. Transactions of the ASAE, St. Joseph, v.31, n.4, p.984-989, 1988.

LAW, S.E. Agricultural electrostatic spray application: a review of significant research and development during de $20^{\text {th }}$ century. Journal Electrostatics, v.51/52, p.25-42, 2001.

MASKI, D.; DIVAKER DURAIRAJ, C.; PUSHPA, T. Characterization of spray liquids for electrostatic charging. Institution of Engineers (Índia), Journal-AG, v.85, p.33-36, 2004.
MASKI, D. Spray chargeability studies and data modeling using electrostatic spraying system for drift mitigation. St. Joseph, Mich: ASAE, 2005. (Paper $n^{\circ}$ 051153).

MATTHEWS, G.A. Electrostatic spraying of pesticides: a review. Crop Protection, v.8, p.3-15, 1989.

RAMOS，H.H.; YANAI，K.; CORREA, I.M.; BASSANESSI, R.B.; GARCIA, L.C. Características da pulverização em citros em função do volume de calda aplicado com turbopulverizador. Engenharia Agrícola, Jaboticabal, v.27, p.56-65, 2007.

SERRA, M.E.; CHAIM, A.; RAETANO, C.G. Pontas de pulverização e eletrificação das gotas na deposição da calda em plantas de crisântemo. Pesquisa Agropecuária Brasileira, v.43, n.4, p.479-485, 2008.

SCHRODER, E.P.; LOECK, A.E. Avaliação do sistema de pulverização eletrostática aérea na redução do volume de calda e dosagem do herbicida glifosate, Revista Brasileira de Agrociência, Pelotas, v.12, n.3, p.319-323, 2006.

SIMMONS, H.C.; LEHTINEN, J.R. Characteristics of electrostatically charged air atomized spray for pesticide application. Pesticide Formulation and Application Systems, v.7, p.116-132, 1986. 